"An Experimental Study of the Effect of Out-of-the-Window Cues on Training of Novice Pilots on a Flight Simulator" by M. Javed Khan, Marcia Rossi, Bruce Heath, S. Firasat Ali, Marcus Ward, Proceedings, Human Factors and Ergonomics Society Conference, San Francisco, California, October 2006, 8 pages. 
Blank Page 


\title{
An Experimental Study of the Effect of Out-of-the-Window Cues on Training Novice Pilots on a Flight Simulator
}

\author{
M. Javed Khan, Marcia Rossi, Bruce Heath, Syed F. Ali, Marcus Ward \\ Tuskegee University, Tuskegee AL 36088
}

\begin{abstract}
The effects of out-of-the-window cues on learning a straight-in landing approach and a level $360^{\circ}$ turn by novice pilots on a flight simulator have been investigated. The treatments consisted of training with and without visual cues as well as density of visual cues. The performance of the participants was then evaluated through similar but more challenging tasks. It was observed that the participants in the landing study who trained with visual cues performed poorly than those who trained without the cues. However the performance of those who trained with a faded-cues sequence performed slightly better than those who trained without visual cues. In the level turn study it was observed that those who trained with the visual cues performed better than those who trained without visual cues. The study also showed that those participants who trained with a lower density of cues performed better than those who trained with a higher density of visual cues.
\end{abstract}

\section{Introduction}

With the increasing availability of low-cost powerful personal computers the development of 'intelligent' flight simulators for use by general aviation flight schools is becoming viable (Ludwig et. al. 2002, Remolina et. al. 2004)). Such simulators will be able to use the available computational power to incorporate not only traditional but also novel training techniques based on learning theories. Also, these simulators will have robust and realistic assessment of task performance to provide feedback thus making selfinstruction effective as well as controlling the training to progress in the right direction. The motivation for developing 'intelligent' flight simulators of course is to reduce the cost of flight training. The FAA already has allowed a certain number of simulator hours on Personal-Computer based Aviation Training Devices (PCATD) to substitute for flight hours thereby reducing the cost of training. However, these simulator hours have to be supervised by a certified flight instructor (CFI). The main advantages of 'intelligent' flight simulators then would be to increase the number of simulator hours substituting for flight hours, effective utilization of simulator hours, increased transfer of training thereby further reducing flight hours to gain proficiency, and reducing/eliminating the need for a CFI during flight simulator training.

To reach these and other such goals there are a number of areas which need to mature before the full potential of such a flight simulator can be realized and used routinely. These include cognitive scaffolds such as aural, visual and environmental cues for learning various flight skills, performance evaluation metrics faithfully capturing certified flight instructors' assessment techniques and seamless incorporation of simulator-based training in the flight training curricula. 
The current research has focused on special out-of -the window (OTW) visual cues in a flight simulator to develop a pedagogical model for training novice pilots. The use of OTW visual cues in flight training is not a novel idea. Two such cues are (a) using a certain perspective of the runway to judge height/glide slope and (b) maintaining a far-off object on a certain location on the windscreen/canopy for learning to maintain glide slope or keeping a tree/fixed point on ground for $360^{\circ}$. level turns. However, if cues are used without understanding their limitations, incorrect habits developed may result in potentially hazardous situations. Foyle et. al. [1992] have pointed to a number of such cues which have high saliency but low reliability as is pertinently pointed out in AIM Chapter 8-1-5 as well.

A number of studies have evaluated various aspects of 'perspective displays or the more commonly referred to as tunnel-in-the-sky concepts. These include the effects of egocentric and exocentric depictions, terrain texture, projected path cues, trajectory shape (Mulder 2003(1), Mulder 2003(2), Doherty \& Wickens 2001, Sachs 2003). Such perspective displays are now commercially available. However it must be noted that this concept is being implemented primarily in a heads-down display (HDD) mode. It has been reported that flying in the tunnel resulted in definite improvements in flight path control. Limited research has been conducted in evaluating its utility as a navigation/flight path aid in a heads-up display (HUD) mode. Fadden et. al.[2001] have reported their observations of the implementation of the 'tunnel-in-the-sky' or 'perspective' displays as a HUD. They determined that it did result in improvements albeit with cognitive tunneling. This was exhibited in the form of inattention towards unexpected traffic outside the tunnel. They also conducted experiments to compare the HUD 'tunnel' with a HDD 'tunnel'. They determined that a HUD implementation had slightly degraded performance in vertical path control while resulting in better performance in lateral flight path control in comparison to a HDD implementation. However their overall assessment was that a HUD implementation would combine the advantage of a HUD and a perspective display.

The advantages of the tunnel-in-the-sky motivated us to investigate a HUD implementation of such a visual cue as a cognitive scaffold to help learn flight path control by novice pilots. In fact such an implementation exists in the form of the 'magic hoops' of the landing lesson in the various versions of the Microsoft Flight Simulator (MSFS).

\section{Experimental Method}

The two maneuvers chosen to evaluate the concept were a straight-in landing approach and a $360^{\circ}$. level turn. These two maneuvers were expected to capture the effects if any of path curvature for such a training concept. Each maneuver was flown by a different batch of novice pilots. Each batch consisted of a control group and a number of treatment groups. The treatments philosophy was based on the concept of gradually reducing the dependence on the scaffold. Two methods of fading were evaluated. The first method was to reduce the number of hoops (tunnel cross section outlines) as the training progressed. The other approach was to mix flights with hoops and without hoops in a certain sequence as the training progressed. Preliminary studies with the hoop- 
reduction technique for a level turn exhibited the need for a certain minimum number of hoops on the flight path for it to be effective as a training aid. For the level-turn maneuver two hoop-densities were used to investigate the effectiveness of hoop density. The study then concentrated on evaluating the effectiveness of mixing flights with hoops and without hoops.

The study was divided into two parts. The first part evaluated the effect of visual cues on a learning a straight-in landing approach, while the other part investigated a level turn. The participants were undergraduate students of an introductory psychology course who took part in the study for extra credit. They had no prior flight simulator or flight experience. The participants were screened for their aptitude to fly the simulator based on their performance in three straight and level flights. The selected 'novice' pilots were then randomly assigned to various treatment and control groups.

\section{Straight-in Landing Approach}

The study consisted of three groups flying a straight-in landing approach in a Cessna 172. Each group flew 8 training flights followed by an evaluation flight more challenging than the training flight. The training flights were flown according to the sequence given in Table I. The participants then flew a straight-in approach with mild turbulence increasing their workload to maintain the glide slope.

\section{$\underline{360} \div$ Level Turn}

In this experiment the participants flew 8 training flights which consisted on a $360^{\circ}$. level turn with a $10^{\circ}$. bank angle. The control group was not provided any cues while the two treatment groups flew some flights with visual cues and others without. The visual cue densities differed for the two treatment groups. The training flights sequence is given in Table II. After the training the participants then flew a more challenging flight by executing a $30^{\circ}$ bank level turn.

\section{Performance Measures}

Various performance measures have been suggested to evaluate a pilot's performance based on flight recorder data (Rantanen et al. 2001, Rantanen \& Johnson 2005). These include standard deviation, root mean square error, number of deviations, total time outside tolerance and mean time to exceed tolerance. In this study the root mean square error (RMSE) was used as a performance measure. In case of the straight-in landing approach, the sum of the RMSE in air speed, rate of descent and runway alignment was used for evaluating performance. For the level turn, RMSE from the required path as evidenced by bank angle and altitude loss determined the performance of the participants. The performance of all participants in each group was averaged to compare between group performances.

\section{Experimental Setup}

The experimental setup was a Microsoft Flight Simulator (MSFS) 2002 environment running on four PCs networked using the IPX/SPX gaming protocol. Three out-of-the-window views were driven by the three PCs while the fourth computer was used to display the synthetic instrument panel. Cougar a joystick and throttle and $\mathrm{CH} 4$ 
rudder pedals were used for controls. Flight parameters of interest were written to file every second for later analysis. The landing approach study used the canned landing lesson in MSFS2002 suitably modified to remove instructor audio and control inputs. The level turn study used gmax to render the visual cue hoops along the turn path. These hoops were included as add-on scenery in MSFS 2002. The flights were designed to start at the same location in a trimmed condition.

\section{Results \& Discussion} visual cues.

The study revealed the following aspects of the influence of out-the-window

\section{Straight-in Landing Approach Task}

A summary of the task performance is shown in Table III. As can be noted, providing visual cues throughout the training regimen (H-group) did not help the participant in learning to fly the simulator. This is primarily because the participants probably were more concerned about flying through the hoops and hence looking OTW rather than also scanning the instrument panel to ensure that the flight parameters for the task were being maintained. In case of the participants who trained without the visual cues (NH-group) performed much better as they had probably learned to divide attention between the OTW view so as to maintain runway alignment as well as scan the instrument panel to maintain the flight parameters for the task. The participants who trained using a mix of the flights with and without visual cues (FH-group) though in the beginning exhibited similar performance as the $\mathrm{H}$-group were most probably able to learn to divide attention effectively and maintain the flight parameters and took the hoops as a challenge to fly through.

\section{Level $360^{\circ}-$ Turn}

The performance in the level turning task is summarized in Table IV. It can be observed that visual cues had a major influence on the performance in the evaluation flight. The performance improved for both the cue-density methods as compared to the group who trained without visual cues. This observation is consistent with the observation of the landing study. The performance was observed to be better for the lower density method as compared to the higher density method. This is attributed to the increased time between hoops thereby forcing the participant to look inside the cockpit at the flight parameters required for the task. With the increased hoops density the tendency to 'chase' the hoops and not paying enough attention to the flight parameters most probably was the cause for the relatively lower performance.

\section{Table I: Treatment Sequences: Straight-in Landing Approach.}

H: Flight with Hoops; NH: Flight with No Hoops; Evaluation Flight: E

\begin{tabular}{|l|l|l|}
\hline $\begin{array}{l}\text { Group\#1 (Control) } \\
(\mathrm{NH})\end{array}$ & Group\#2 (H) & Group\#3 (FH) \\
\hline $\mathrm{NH}$ & $\mathrm{H}$ & $\mathrm{H}$ \\
\hline $\mathrm{NH}$ & $\mathrm{H}$ & $\mathrm{H}$ \\
\hline
\end{tabular}




\begin{tabular}{|l|l|l|}
\hline $\mathrm{NH}$ & $\mathrm{H}$ & $\mathrm{H}$ \\
\hline $\mathrm{NH}$ & $\mathrm{H}$ & $\mathrm{NH}$ \\
\hline $\mathrm{NH}$ & $\mathrm{H}$ & $\mathrm{NH}$ \\
\hline $\mathrm{NH}$ & $\mathrm{H}$ & $\mathrm{H}$ \\
\hline $\mathrm{NH}$ & $\mathrm{H}$ & $\mathrm{H}$ \\
\hline $\mathrm{NH}$ & $\mathrm{H}$ & $\mathrm{NH}$ \\
\hline $\mathrm{E}$ & $\mathrm{E}$ & $\mathrm{E}$ \\
\hline
\end{tabular}

Table II: $\underline{\text { Treatment Sequences for a } 360} \stackrel{\text { Level Turn }}{\text { Len }}$

H: Flight with Hoops; NH: Flight with No Hoops; Evaluation Flight: E

\begin{tabular}{|l|l|l|}
\hline Group\#1 (Control) & Group\#2 (36 Hoops) & Group\#3 (18 Hoops) \\
\hline NH & H & H \\
\hline NH & N & N \\
\hline NH & H & H \\
\hline NH & N & N \\
\hline NH & N & N \\
\hline NH & H & H \\
\hline NH & N & N \\
\hline NH & N & N \\
\hline NH & N & N \\
\hline E & E & E \\
\hline
\end{tabular}

Table III: Composite Error for Straight-in Landing Approach

\begin{tabular}{|l|l|}
\hline Group & Error \\
\hline Control (No hoops) & 25.5 \\
\hline All training flights with hoops (H-group) & 32.1 \\
\hline Faded-cues training flights (FH-group) & 22.2 \\
\hline
\end{tabular}

Table IV: Error for Level $360^{\circ}-\underline{\text { Turn }}$

\begin{tabular}{|l|l|l|}
\hline Group & Error in Bank Angle & Error in Altitude \\
\hline Control (No Hoops) & 17.99 & 233.1 \\
\hline Low Density Hoops & 10.12 & 109.5 \\
\hline High Density Hoops & 11.22 & 170.3 \\
\hline
\end{tabular}

\section{References:}

Emilio Remolina, Sowmya Ramachandran, Daniel Fu, Richard Stottler and William R. Howse [2004], Intelligent Simulation-Based Tutor for Flight Training, Interservice/Industry Training, Simulation, and Education Conference (I/ITSEC) 2004 2004 Paper No. 1743 Page 1 of 13 
Fadden S., Ververs P.M., Wickens C.D., [2001]"Pathway HUDs: Are they Viable?", Human Factors, 43(2), Summer, 2001

Foyle, D.C., Kaiser, M.K. and Johnson, W.W. [1992]. Visual Cues in Low-level Flight: Implications for Pilotage, Training, Simulation, and Enhanced/Synthetic Vision Systems, American Helicopter Society 48th Annual Forum, Vol. 1, 253-260.

Jeremy Ludwig, Sowmya Ramachandran, William Howse [2002], Developing an Adaptive Intelligent Flight Trainer, Stottler Henke Associates, San Mateo, CA .http://www.stottlerhenke.com/papers/IITSEC-02-IFT.pdf. accessed on $02 / 23 / 2006$

J. B. McKinley, E. Heidhausen, J. A. Cramer and N. J. Krone, Flight Testing of an Airborne SVS With Highway-In-The-Sky on a Head-Up Display http://www.urf.com/madl/papers/DASC-svs-hits.pdf accessed on 02/23/2006.

Lisa C Thomas and Christopher D Wickens [2004], Eye-Tracking and Individual Differences in Off-Normal Event Detection when Flying with a Synthetic Vision System Display, Proceedings of the Human Factors and Ergonomics Society 48th Annual Meeting. Santa Monica: Human Factors and Ergonomics Society, 2004. http://www.humanfactors.uiuc.edu/Reports\&PapersPDFs/humfac04/thom wick.pdf. Accessed on 02/23/06

FAA AC 61-126 Appendix 1, C \& FAR 61.4(c) 